\title{
A density functional study on equilibrium geometries, stabilities and electronic properties of $\mathrm{Au}_{5} \mathrm{Li}$ binary clusters
}

\author{
Ajanta Deka $\cdot$ Ramesh Chandra Deka
}

Received: 30 November 2011 / Accepted: 13 March 2012/Published online: 29 March 2012

(C) The Author(s) 2012. This article is published with open access at Springerlink.com

\begin{abstract}
All electron scalar relativistic calculations have been performed to investigate the electronic structures of isomeric $\mathrm{Au}_{5} \mathrm{Li}$ binary clusters at the BLYP/DNP level of theory. The properties of these clusters are compared with the pristine $\mathrm{Au}_{6}$ clusters. As in case of the pure $\mathrm{Au}_{6}$ cluster, the minimum energy structure of the bimetallic $\mathrm{Au}_{5} \mathrm{Li}$ cluster is triangular with the lithium atom at the mid position. It is found that substitution of a gold atom by a lithium atom in the $\mathrm{Au}_{6}$ clusters leads to an increase in the binding energy per atom, the HOMO-LUMO gap and the chemical hardness of the structures. Thus, lithium substitution leads to stabilization of the $\mathrm{Au}_{6}$ clusters. Further, the response of various sites of the minimum-energy triangular $\mathrm{Au}_{5} \mathrm{Li}$ and $\mathrm{Au}_{6}$ clusters towards impending electrophilic and nucleophilic attacks has been determined using DFTbased local reactivity descriptors. It is found that lithium substitution leads to an increase in the number of sites prone to attack by nucleophiles like $\mathrm{CO}$ or $\mathrm{H}_{2} \mathrm{O}$.
\end{abstract}

Keywords Binary clusters - Lithium substitution · Density functional theory $\cdot$ Relative electrophilicity . Relative nucleophilicity

\footnotetext{
A. Deka

Department of Physics, Girijananda Chowdhury Institute of Management and Technology, Hatkhowapara, Azara, Guwahati 781017, India

R. C. Deka ( $₫)$

Department of Chemical Sciences, Tezpur University,

Napaam, Tezpur 784028, Assam, India

e-mail: ramesh@tezu.ernet.in
}

\section{Introduction}

The study of metal clusters is interesting as they provide us an opportunity to examine the size evolution of metals from the atomic to the bulk state. The properties of a material at the nanoscale can be very different from that of the bulk. As nanoclusters contain a large number of low coordinated surface atoms they readily form bonds with other species, and hence, can be very useful as catalysts. Among metal clusters, gold has been very widely investigated due to its applications in a number of fields like catalysis (Pyykkö 2004) biological diagnostics, medicine, molecular electronic devices (Cui et al. 2001; Valden et al. 1998), nanostructured materials (Whetten et al. 1996; Andres et al. 1996), optics, material science and solid-state chemistry. The properties of pristine gold clusters can be altered by doping the cluster with impurities. This tailoring of the cluster composition leads to changes in the structural, electronic, magnetic and reactivity properties, thus increasing their potential applications. The modification of cluster properties by doping can be effected in two dimensions, viz, by varying the nature and amount of the dopant. A number of studies have been carried out on doped gold clusters (Majumder et al. 2006; David et al. 2010), the most common elements used for doping being the transition metals ( $\mathrm{Li}$ et al. 2005). There are also studies on the doping of gold clusters by $s$ and $p$ block elements. The electronic structure of alkali metals is similar to that of gold as both contain a single $s$ valence electron. This makes the study of alkali-metal-doped gold clusters particularly interesting. Studies on gold aurides have revealed that the gold-alkali metal bond is highly polar with a large charge transfer from the alkali atom to the gold. Due to the high difference in electronegativity between the alkali metals and gold, alkali aurides are found to be highly stable.

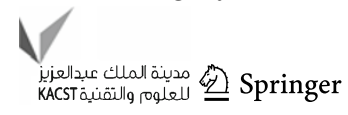


Among the alkali metals, the electronic structure of lithium is different as it presents an unfilled $p$ shell (Rajesh and Majumder 2009; Heiz and Bullock 2004). In addition, lithium is the lightest element that is metallic under normal conditions. So it would be interesting to investigate how doping of a gold cluster by a lithium atom modifies its electronic properties and reactivity.

In an earlier study on the structural and electronic properties of $\mathrm{Au}_{n}$ clusters $(n=2-13)$ using DFT (Deka and Deka 2008) we have found that the triangular $\mathrm{Au}_{6}$ cluster is highly stable. A number of isomeric $\mathrm{Au}_{6}$ clusters were considered in that study. In this paper, we examine the effect of doping $\mathrm{Au}_{6}$ clusters with a lithium atom resulting in the formation of $\mathrm{Au}_{5} \mathrm{Li}$ clusters. An exhaustive search for enhanced catalytic properties of nanoclusters by doping can be accomplished only if all isomers of the parent cluster are considered. So, we have taken a number of isomeric structures of the highly stable triangular $\mathrm{Au}_{6}$ cluster from our earlier study as candidate structures for the present investigation.

\section{Computational details}

Density functional calculations were performed to obtain full geometry optimization at the level of a generalized gradient approach via the BLYP exchange-correlation functional. This functional incorporates Becke's exchange (Becke 1988) and Lee-Yang-Parr correlation (Lee et al. 1988). All calculations were performed using the $\mathrm{DMol}^{3}$ suite of programs (Delley 1990). Scalar relativistic effects (VPSR) (Delley 1998) were incorporated into our all electron calculations to account for gold, which is a heavy atom. Relativistic effects in gold lead to radial contraction and energetic stabilization of the $s$ and $p$ orbitals and a consequent radial expansion and energetic destabilization of the $d$ and $f$ orbitals. This leads to stronger interaction between the valence $5 d$ and $6 s$ orbitals thus reducing the $s-$ $d$ energy gap. Relativistic effects lead to shorter metalmetal bonds, and hence, more stable clusters. The planarity of gold clusters up to large system size is attributed to relativistic effects. The effects of relativity on properties like bond length, ionization potential, electron affinity, dissociation energy, etc. for $\mathrm{Au}_{2}, \mathrm{AuH}$ and $\mathrm{Au}$ (solid) has been listed by Pyykkö (2004) in his excellent review on gold clusters.

Self consistent field procedures were carried out with convergence criteria of $1 \times 10^{-5}$ a.u. on the total energy and $1 \times 10^{-6}$ a.u. on the electron density. The basis set used is of double numeric quality with polarization functions (DNP) (Delley and Ellis 1982). This basis set is comparable with the Gaussian $6-31 G^{* *}$ basis set, but the DNP basis set is supposed to be more accurate than a
Gaussian basis set of similar size. All calculations are spin restricted and the geometry optimizations have been performed without imposing any symmetry constraint.

The average binding energy per atom is computed from:

$E_{\mathrm{b}}=\left[5 E\left(\mathrm{Au}_{1}\right)+E\left(\mathrm{Li}_{1}\right)-E\left(\mathrm{Au}_{5} \mathrm{Li}\right)\right] / 6$

where $E\left(\mathrm{Au}_{5} \mathrm{Li}\right)$ is the energy of the $\mathrm{Au}_{5} \mathrm{Li}$ cluster.

Chemical hardness is used as a parameter to determine the reactivity of a system. In density functional theory, hardness $(\eta)$ of an electronic system is defined as the second derivative of energy $(E)$ with respect to the number of electrons $(N)$ at constant external potential, $v(\vec{r})$ (Fukui 1982).

$\eta=\frac{1}{2}\left(\frac{\partial^{2} E}{\partial N^{2}}\right)_{v(\vec{r})}=\frac{1}{2}\left(\frac{\partial \mu}{\partial N}\right)_{v(\vec{r})}$

where $\mu$ is the chemical potential of the system.

Global hardness, $\eta$ can be approximated as

$\eta=\frac{\mathrm{LUMO}-\mathrm{HOMO}}{2}$

Fukui function $f(\vec{r})$, as defined by Parr and Yang (Parr and Yang 1984; Yang et al. 1984), is a mixed second derivative of energy of the system with respect to the number of electrons $N$ and constant potential $v(\vec{r})$ :

$f(\vec{r})=\left(\frac{\partial^{2} E}{\partial N \partial v(\vec{r})}\right)=\left[\frac{\delta \mu}{\delta v(\vec{r})}\right]_{N}=\left(\frac{\partial \rho(\vec{r})}{\partial N}\right)_{v(\vec{r})}$

where $\rho(\vec{r})$ represents the electron density at position $\vec{r}$ of the chemical species.

Fukui function expresses the sensitivity of the chemical potential of a system to an external perturbation. Higher the value of Fukui function at a particular site, more is its reactivity. The condensed Fukui function for an atom $k$ in a molecule having $N$ electrons can be obtained from the finite difference approximation as:

$f_{k}^{+}=\left[q_{k}(N+1)-q_{k}(N)\right]$ for nucleophilic attack

and

$f_{k}^{-}=\left[q_{k}(N)-q_{k}(N-1)\right]$ for electrophilic attack

where $q_{k}(N), q_{k}(N+1)$ and $q_{k}(N-1)$ are electronic populations on the particular atom $k$ of the molecule with $N, N+1$ and $N-1$ electrons, respectively. The 'relative electrophilicity' and 'relative nucleophilicity' values for the particular atom can be obtained from the ratio of Fukui functions $f_{k}^{+} / f_{k}^{-}$and $f_{k}^{-} / f_{k}^{+}$, respectively.

A large number of $\mathrm{Au}_{6}$ isomeric clusters were used as candidate structures. One atom of the pristine gold cluster was replaced by a lithium atom and then the geometry was fully optimized. This replacement by a lithium atom was done for each unique atom of the cluster. Vibrational frequency calculations were then carried out at the optimized 
geometry to verify that the obtained structures are global minima on the potential energy surface. The Fukui functions were calculated on the basis of Hirshfeld population analysis.

\section{Results and discussion}

We started with eight isomeric structures of the $\mathrm{Au}_{6}$ cluster and doped each unique atom of each structure by a lithium atom. These isomeric structures are shown in Fig. 1. These clusters have been arranged in the order of increasing energy. The minimum energy structure is triangular. These structures have been taken from our earlier work on the structural and electronic properties of stable $\mathrm{Au}_{n}$ ( $n=2-13$ ) clusters (Deka and Deka 2008). Substitution of a gold atom by a lithium atom resulted in ten stable structures of the $\mathrm{Au}_{5} \mathrm{Li}$ cluster. The other geometries either did not converge or exhibited negative frequencies. The optimized geometries are shown in Fig. 2.

The structures have been named as $S_{1}, S_{2}, S_{3}, \ldots, S_{10}$ by their relative stability, namely, $S_{1}$ is the most stable structure and $\mathrm{S}_{10}$ is the least stable. Of the ten structures of the $\mathrm{Au}_{5} \mathrm{Li}$ cluster, five are found to be planar. The structures of the $\mathrm{Au}_{6}$ clusters are mostly retained on doping with a lithium atom, the two-triangle $\mathrm{S}_{8}$ structure being an exception. In case of the $\mathrm{Au}_{6}$ cluster, this structure is threedimensional having $\mathrm{C}_{2 \mathrm{~h}}$ symmetry, while in case of $\mathrm{Au}_{5} \mathrm{Li}$, it is planar. The structure $\mathrm{S}_{2}$ which is a capped square pyramid has not been found in case of the $\mathrm{Au}_{6}$ cluster. As in case of pristine $\mathrm{Au}_{6}$ cluster, the minimum energy structure of the $\mathrm{Au}_{5} \mathrm{Li}$ cluster is triangular with a small inner triangle housed inside a big outer triangle. The lithium atom occupies the mid position which forms a vertex of the inner triangle. The other stable isomer having a similar triangular structure is $\mathrm{S}_{4}$ where the lithium atom occupies the apex position forming a vertex of the outer triangle. To the best of our knowledge there has been no theoretical or experimental study on the $\mathrm{Au}_{5} \mathrm{Li}$ bimetallic cluster. So, a direct comparison of our parameters with those of other workers is not possible. As seen from Table 1, the average $\mathrm{Au}-\mathrm{Au}$ distances for the various isomers of the pristine gold clusters lie in the range 2.63$2.79 \AA$ while those for the $\mathrm{Au}_{5} \mathrm{Li}$ clusters vary between 2.45 and $2.60 \AA$, respectively. Another DFT study with the PW91 exchange correlation functional and a plane wave basis set ( $\mathrm{Li}$ et al. 2006) gives average $\mathrm{Au}-\mathrm{Au}$ distances varying between 2.58 and $2.82 \AA$ for various isomers of pristine $\mathrm{Au}_{6}$ clusters. Our study reveals that bond distances for neighbouring $\mathrm{Au}-\mathrm{Li}$ atom pairs are shorter than those of the $\mathrm{Au}-\mathrm{Au}$ atom pairs, indicating that $\mathrm{Li}$ substitution leads to stronger bonds. However, our $\mathrm{Au}-\mathrm{Li}$ distances are much larger compared to the AuLi dimer bond length (2.27 ̊) (Leonardo et al. 2006), and hence, much weaker.

The binding energy per atom values of the different isomers of the $\mathrm{Au}_{5} \mathrm{Li}$ cluster is given in Table 1. In addition, shown in Table 1 are the binding energies per atom of the corresponding pristine $\mathrm{Au}_{6}$ clusters for comparison. The most stable triangular $\mathrm{Au}_{5} \mathrm{Li}$ cluster $\mathrm{S}_{1}$ with the lithium atom having a coordination number of 4 exhibits the highest binding energy of $1.966 \mathrm{eV}$. The binding energy per atom of the pristine triangular $\mathrm{Au}_{6}$ cluster has a value
Fig. 1 Optimized geometries of $\mathrm{Au}_{6}$ clusters arranged in the order of increasing energy. The unique atoms have been marked

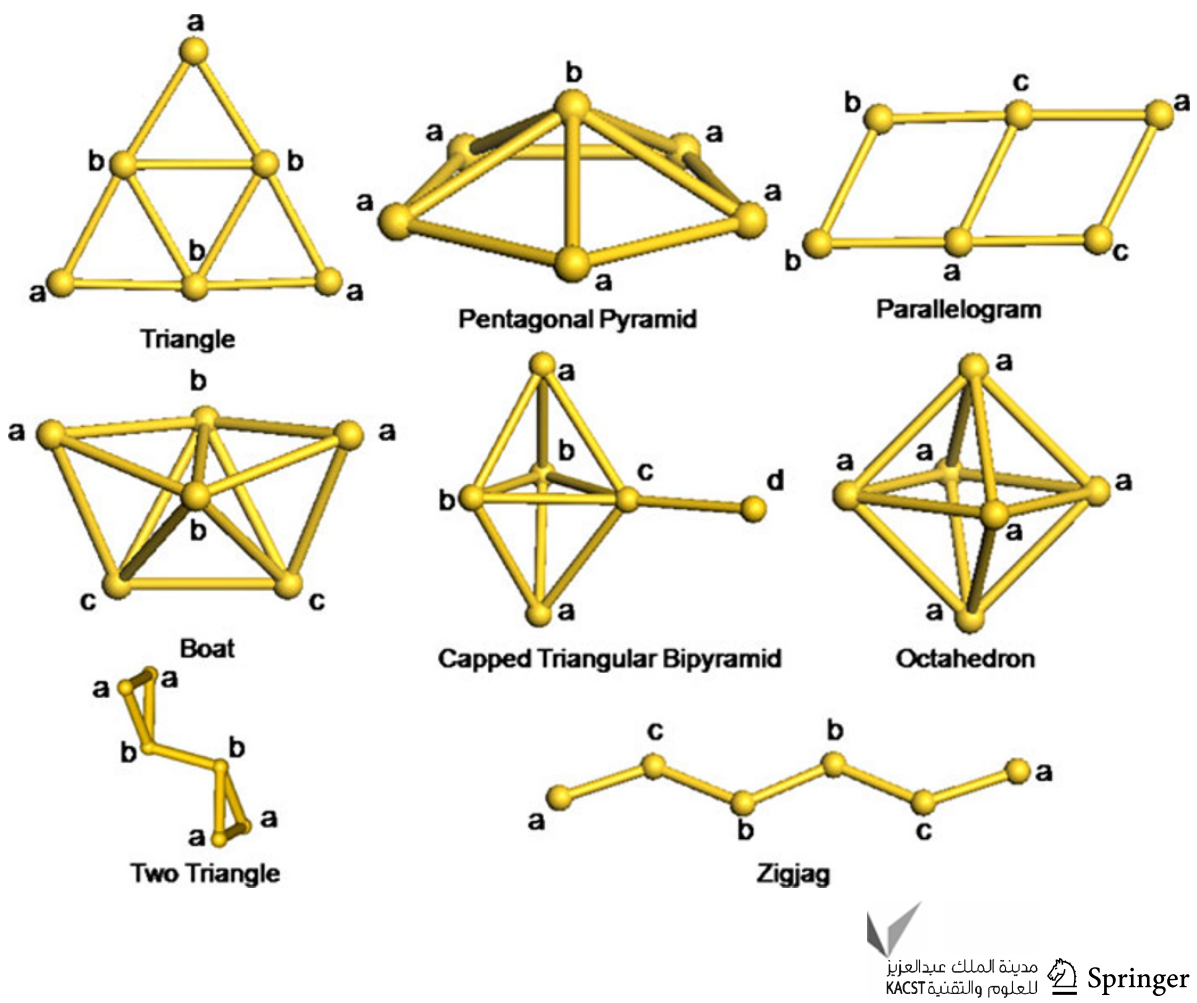


Fig. 2 Isomeric structures of $\mathrm{Au}_{5} \mathrm{Li}$ clusters arranged in the order of increasing energy. Gold atoms are numbered as 1 and $\mathrm{Li}$ atoms as 2 . The relative energies are given in $\mathrm{eV}$

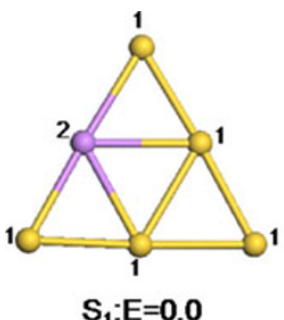

$S_{1}: E=0.0$

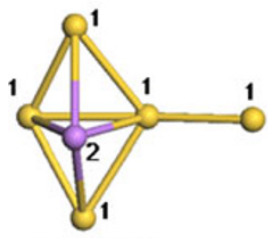

$S_{5}: E=0.76$

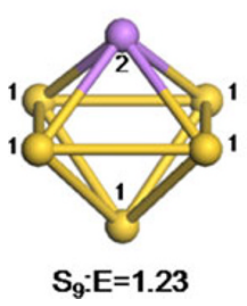

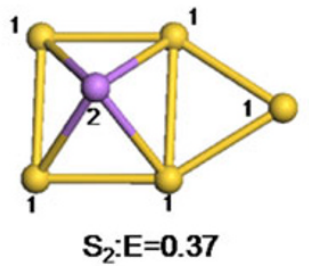
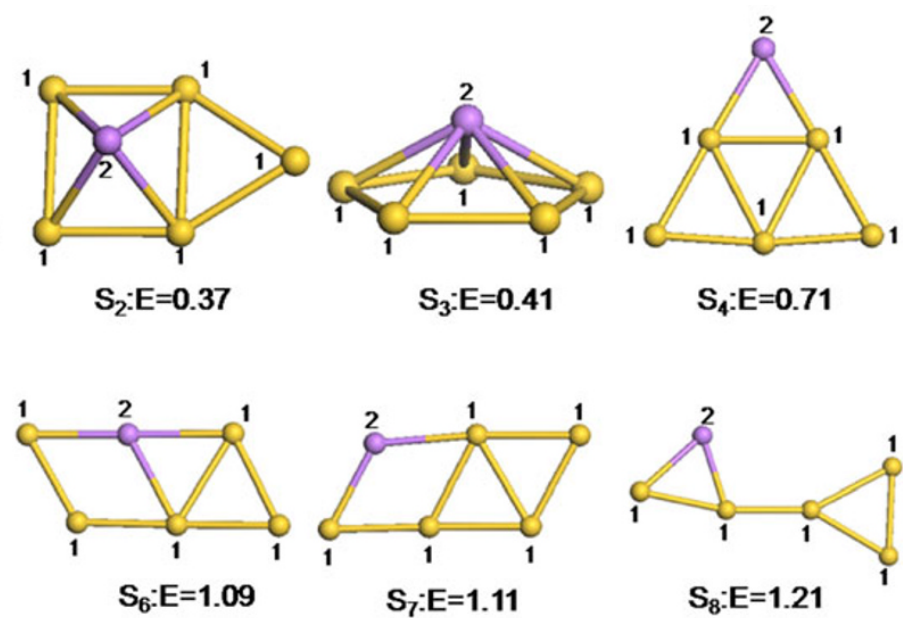

$S_{8}: E=1.21$

Table 1 Geometric and energetic parameters of pristine $\mathrm{Au}_{6}$ and bimetallic $\mathrm{Au}_{5} \mathrm{Li}$ clusters

\begin{tabular}{|c|c|c|c|c|c|c|c|c|c|}
\hline \multirow[t]{2}{*}{ Isomer } & \multirow[t]{2}{*}{ Relative energy } & \multirow[t]{2}{*}{$\mathrm{Au}-\mathrm{Au}$} & \multirow[t]{2}{*}{$\mathrm{Au}-\mathrm{Li}$} & \multicolumn{2}{|l|}{$E_{\mathrm{b}}$} & \multicolumn{2}{|c|}{ H-L gap } & \multicolumn{2}{|l|}{$\eta$} \\
\hline & & & & $\mathrm{Au}_{5} \mathrm{Li}$ & $\mathrm{Au}_{6}$ & $\mathrm{Au}_{5} \mathrm{Li}$ & $\mathrm{Au}_{6}$ & $\mathrm{Au}_{5} \mathrm{Li}$ & $\mathrm{Au}_{6}$ \\
\hline $\mathrm{S}_{1}$ & 0.000 & 2.631 & 2.645 & 1.966 & 1.843 & 1.736 & 1.727 & 0.868 & 0.864 \\
\hline $\mathrm{S}_{2}$ & 0.369 & 2.696 & 2.602 & 1.905 & & 1.263 & & 0.632 & \\
\hline $\mathrm{S}_{3}$ & 0.410 & 2.664 & 2.646 & 1.898 & 1.690 & 1.684 & 1.641 & 0.842 & 0.821 \\
\hline $\mathrm{S}_{4}$ & 0.709 & 2.703 & 2.577 & 1.848 & 1.843 & 1.586 & 1.727 & 0.793 & 0.864 \\
\hline $\mathrm{S}_{5}$ & 0.761 & 2.686 & 2.645 & 1.839 & 1.588 & 1.400 & 0.630 & 0.700 & 0.315 \\
\hline $\mathrm{S}_{6}$ & 1.090 & 2.649 & 2.656 & 1.785 & 1.676 & 0.322 & 0.176 & 0.161 & 0.088 \\
\hline $\mathrm{S}_{7}$ & 1.106 & 2.677 & 2.597 & 1.782 & 1.676 & 0.193 & 0.176 & 0.096 & 0.088 \\
\hline $\mathrm{S}_{8}$ & 1.209 & 2.639 & 2.451 & 1.765 & & 0.735 & & 0.368 & \\
\hline $\mathrm{S}_{9}$ & 1.225 & 2.783 & 2.572 & 1.762 & 1.702 & 0.721 & 1.806 & 0.361 & 0.903 \\
\hline $\mathrm{S}_{10}$ & 1.914 & 2.785 & 2.685 & 1.647 & 1.588 & 0.367 & 0.630 & 0.184 & 0.315 \\
\hline
\end{tabular}

Bond lengths are in $\AA$ and energies are in $\mathrm{eV}$

of $1.843 \mathrm{eV}$. Similar GGA calculations at the PW91PW91/ 6-31G* (Majumder et al. 2006) and PW91/LANL2DZ (Li et al. 2007) levels of theory reveal binding energy per atom values of 1.82 and $1.826 \mathrm{eV}$, respectively, for the minimum energy triangular $\mathrm{Au}_{6}$ cluster. Our binding energy value for triangular $\mathrm{Au}_{5} \mathrm{Li}$ cluster is higher than each of these values. Thus, lithium substitution leads to an increase in the binding energy per atom of this structure by $0.123 \mathrm{eV}$ in our case. For the structure $\mathrm{S}_{4}$ where the lithium atom has a coordination number of 2 , the binding energy per atom value is $1.848 \mathrm{eV}$. As can be seen from Table 1 this value is only slightly higher than that of the corresponding pure gold cluster. Thus, gain in binding energy due to lithium substitution into the minimum energy triangular $\mathrm{Au}_{6}$ cluster is more when the lithium atom has a higher coordination number. On going from the most stable $S_{1}$ cluster to the least stable $S_{10}$ cluster the binding energies go on decreasing. The $S_{10}$ structure has a binding energy of $1.647 \mathrm{eV}$. A comparison of the binding energy values of the $\mathrm{Au}_{5} \mathrm{Li}$ and $\mathrm{Au}_{6}$ clusters reveal that in each case the binding energies increase due to lithium substitution.

The HOMO-LUMO gap is a measure of stability of clusters. Larger the HOMO-LUMO gap, more stable is the cluster. In Table 1 we show the HOMO-LUMO gap of the optimized $\mathrm{Au}_{5} \mathrm{Li}$ clusters along with those of the corresponding pure $\mathrm{Au}_{6}$ clusters. The most stable $\mathrm{Au}_{5} \mathrm{Li}$ cluster $\mathrm{S}_{1}$ 
exhibits the highest HOMO-LUMO gap of $1.736 \mathrm{eV}$. The other triangular $\mathrm{Au}_{5} \mathrm{Li}$ cluster $\mathrm{S}_{4}$ has a HOMO-LUMO gap of $1.586 \mathrm{eV}$. The parent structure of both these clusters is the triangular $\mathrm{Au}_{6}$ cluster having a HOMO-LUMO gap of $1.727 \mathrm{eV}$. Thus, lithium substitution leads to increase in the LUMO-LUMO gap, the change in HOMO-LUMO gap being larger when lithium occupies the mid position. The pentagonal pyramidal structure $S_{3}$ with a 5-coordinated lithium atom at the top also has a large HOMO-LUMO gap of $1.684 \mathrm{eV}$. The analogous $\mathrm{Au}_{6}$ cluster has an energy gap of $1.641 \mathrm{eV}$. It is seen that in most cases lithium substitution leads to an increase in the HOMO-LUMO gap with the octahedral structure $\mathrm{S}_{9}$ being an exception. In addition, shown in Table 1 are the values of chemical hardness of the clusters. As expected, the most stable cluster has the highest value of chemical hardness. It is seen that for most of the structures, chemical hardness increases due to lithium substitution.

To study the local reactivity at different sites within a cluster, we choose the most stable $\mathrm{Au}_{5} \mathrm{Li}$ cluster, $\mathrm{S}_{1}$ for our investigation. The Hirshfeld charges and Fukui functions for this structure are shown in Table 2. In addition, shown are analogous values of the pristine triangular $\mathrm{Au}_{6}$ cluster. As can be seen from the table, the lithium atom bears a positive charge of $0.189 \mathrm{q}$ while the gold atoms are negatively charged. In case of the pure $\mathrm{Au}_{6}$ cluster, three atoms, namely, those lying at the vertices of the outer triangle carry negative charges while those forming the vertex of the inner triangle bear positive charges of equal magnitude, thus making the cluster neutral as a whole. Thus, on doping the $\mathrm{Au}_{6}$ cluster with the lithium atom, lithium donates electrons to the gold atoms becoming positively charged in the process, while the negative charge on the gold atoms increase. In addition, shown in Table 2 are the values of the Fukui functions at different sites within the cluster. For the pure $\mathrm{Au}_{6}$ cluster the atoms lying at the vertex of the outer triangle have higher values of relative electrophilicity $f^{+} / f^{-}$indicating that these atoms are prone to attack by nucleophiles like $\mathrm{CO}$. The atoms forming the vertex of the inner triangle on the other hand having higher values of relative nucleophilicity $f^{-} / f^{+}$and hence are prone to attack by electrophiles like $\mathrm{O}_{2}$. On substituting the lithium atom at the mid site the outer gold atoms still remain prone to attacks by nucleophiles like CO. But in addition we have a new site which prefers a nucleophile like $\mathrm{CO}$ or $\mathrm{H}_{2} \mathrm{O}$. A look at Table 2 reveals that the lithium atom has a higher value of $f^{+} / f^{-}$compared to $f^{-} / f^{+}$, and hence, prefers a nucleophile like CO. In case of the pure gold cluster this site is prone to electrophilic attack. Thus, as a result of lithium substitution the number of possible sites for nucleophilic attack on the system increases.

The spatial orientation of the highest occupied molecular orbital (HOMO) and the lowest unoccupied molecular orbital (LUMO) energy levels are depicted in Fig. 3. It is seen that the HOMO is strongly delocalized with contribution from all the gold atoms of the cluster. This
Table 2 Hirshfeld charges, Fukui functions, relative electrophilicity and relative nucleophilicity values for unique atoms of pristine $\mathrm{Au}_{6}$ and bimetallic $\mathrm{Au}_{5} \mathrm{Li}$ clusters

\begin{tabular}{|c|c|c|c|c|c|c|c|c|}
\hline \multirow[t]{2}{*}{ Unique atom } & \multicolumn{4}{|l|}{$\mathrm{Au}_{5} \mathrm{Li}$} & \multicolumn{4}{|l|}{$\mathrm{Au}_{6}$} \\
\hline & $f^{+}$ & $f^{-}$ & $f^{+} / f^{-}$ & Charges & $f^{+}$ & $f^{-}$ & $f^{+} / f^{-}$ & Charges \\
\hline Au1 & 0.216 & 0.203 & 1.064 & -0.013 & 0.232 & 0.197 & 1.178 & 0.0012 \\
\hline $\mathrm{Au} 2$ & 0.216 & 0.203 & 1.064 & -0.081 & 0.232 & 0.197 & 1.178 & 0.0012 \\
\hline Au3 & 0.279 & 0.198 & 1.409 & -0.013 & 0.232 & 0.197 & 1.178 & 0.0012 \\
\hline $\mathrm{Au} 4$ & 0.116 & 0.172 & 0.674 & -0.081 & 0.102 & 0.137 & 0.745 & -0.0011 \\
\hline Li5/Au5 & 0.056 & 0.052 & 1.077 & 0.189 & 0.102 & 0.137 & 0.745 & -0.0011 \\
\hline Au6 & 0.116 & 0.172 & 0.674 & -0.003 & 0.102 & 0.137 & 0.745 & -0.0011 \\
\hline
\end{tabular}

Fig. 3 Spatial orientations of the highest occupied molecular orbital and lowest unoccupied molecular orbital of the minimum energy triangular $\mathrm{Au}_{5} \mathrm{Li}$ cluster

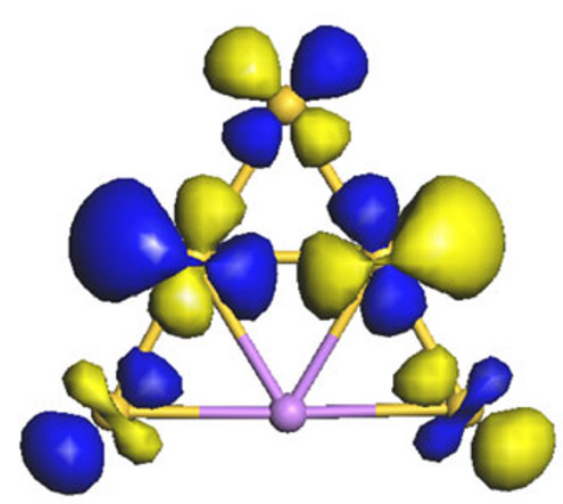

HOMO

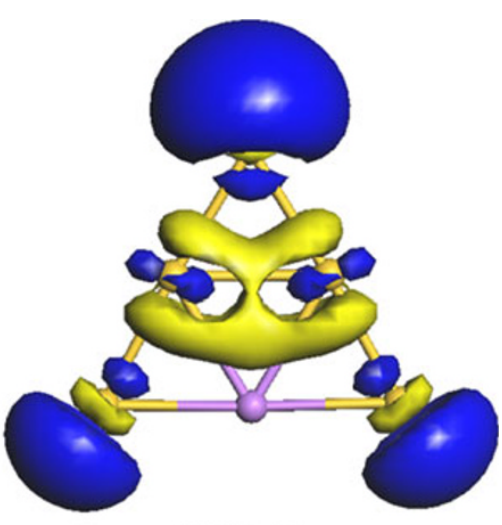

LUMO 
delocalization of the electronic wave-function leads to stabilization of the planar structure. The LUMO is found to be concentrated at the gold atoms forming the vertex of the outer triangle. So the electron accepting power of the cluster is higher at these sites. Thus, an electron-rich species like $\mathrm{CO}$ is most likely to be adsorbed at these sites. This further confirms the predictions regarding nucleophilic attack obtained from the Fukui function values.

\section{Summary}

We have obtained the optimized geometries of ten isomeric $\mathrm{Au}_{5} \mathrm{Li}$ clusters. These structures were obtained by replacing one gold atom of pure $\mathrm{Au}_{6}$ clusters by a lithium atom. The structure and energetics of these clusters were compared with analogous structures of the pristine $\mathrm{Au}_{6}$ cluster. It is found that the structures of most of the gold clusters are retained on lithium substitution. Parameters like binding energy per atom, HOMO-LUMO gap and chemical hardness increase on doping the $\mathrm{Au}_{6}$ cluster by a lithium atom indicating that the bimetallic $\mathrm{Au}_{5} \mathrm{Li}$ clusters are more stable than their pristine $\mathrm{Au}_{6}$ counterparts. Further it is found from Fukui function values that lithium substitution leads to increase in the number of sites prone to be attacked by nucleophiles like CO. The HOMO of the minimum energy planar triangular $\mathrm{Au}_{5} \mathrm{Li}$ cluster is strongly delocalized leading to its high stability.

Acknowledgments The authors thank the Department of Science and Technology, New Delhi for financial support.

Open Access This article is distributed under the terms of the Creative Commons Attribution License which permits any use, distribution, and reproduction in any medium, provided the original author(s) and the source are credited.

\section{References}

Andres RP, Bein T, Dorogi M et al (1996) "Coulomb staircase" at room temperature in a self-assembled molecular nanostructure. Science 272:1323-1325

Becke AD (1988) Density-functional exchange-energy approximation with correct asymptotic behaviour. Phys Rev A 38:3098-3100
Cui XD, Primak A, Zarate X et al (2001) Reproducible measurement of single-molecule conductivity. Science 294:571-574

David J, Guerra D, Hadad CZ, Resprepo A (2010) Structure and reactivity of the ${ }^{1} \mathrm{Au}_{6} \mathrm{Pt}$ clusters. J Phys Chem A 114:10726-10731

Deka A, Deka RC (2008) Structural and electronic properties of stable $\mathrm{Au}_{n}(n=2-13)$ clusters: a density functional study. $\mathbf{J}$ Mol Struct THEOCHEM 870:83-93

Delley B (1990) The all-electron numerical method for solving the local density functional for polyatomic molecules. J Chem Phys 92:508-517

Delley B (1998) A scattering theoretic approach to scalar relativistic corrections on bonding. Int J Quantum Chem 69:423-433

Delley B, Ellis DE (1982) Efficient and accurate expansion methods for molecules in local density models. J Chem Phys 76:1949-1960

Fukui K (1982) Role of frontier orbitals in chemical reactions. Science 218:747-754

Heiz U, Bullock EL (2004) Fundamental aspects of catalysis on supported metal clusters. J Mater Chem 14:564-577

Lee C, Yang W, Parr RG (1988) Development of the Colle-Salvetti correlation-energy formula into a functional of the electron density. Phys Rev B 37:785-789

Leonardo B, Tarantelli F, Sgamellotti A (2006) The electronic structure of alkali aurides: a four-component Dirac-Kohn-Sham study. J Phys Chem A 110:4543-4554

Li X, Kiran B, Cui L-F, Wang L-S (2005) Magnetic properties in transition-metal-doped gold clusters: $M @ \mathrm{Au}_{6}(M=\mathrm{Ti}, \mathrm{V}, \mathrm{Cr})$. Phys Rev Lett 95:23401-1-23401-4

Li X, Tollberg B, Hu X, Wang L (2006) Structural study of gold clusters. J Chem Phys 124:114309-1-114309-10

Li X-B, Wang H-I, Yang X-D, Zhu Z-H (2007) The dependence of the structures and energetic and electronic properties of gold clusters. J Chem Phys 126:084505-1-104504-8

Majumder C, Kandalam AK, Jena P (2006) Structure and bonding of $\mathrm{Au}_{5} M(M=\mathrm{Na}, \mathrm{Mg}, \mathrm{Al}, \mathrm{Si}, \mathrm{P}$, and $\mathrm{S})$ clusters Phys Rev. B 74:205437-1-205437-6

Parr RG, Yang WT (1984) Density functional approach to the frontier-electron theory of chemical reactivity. J Am Chem Soc 106:4049-4050

Pyykkö P (2004) Theoretical chemistry of gold. Angew Chem Int Ed 43:4412-4456 (And references therein)

Rajesh C, Majumder C (2009) Oxidation of Al doped Au clusters: a first principles study. J Chem Phys 130:234309

Valden M, Lai X, Goodman DW (1998) Onset of catalytic activity of gold on titania with the appearance of nonmetallic properties. Science 281:1647-1650

Whetten RL, Khoury JT, Alvarez MM et al (1996) Nanocrystal gold molecules. Adv Mat 8:428-433

Yang W, Parr RG, Pucci R (1984) Electron-density, Kohn-Sham frontier orbitals, and Fukui functions. J Chem Phys 81:2862-2863 